\title{
Pain During Venous Cannulation
}

\author{
A Randomized Controlled Study of the Efficacy of Local Anesthetics
}

Dirk Rüsch, Tilo Koch, Markus Spies, Leopold HJ. Eberhart

\section{SUMMARY}

Background: The pain of venous puncture for the cannulation of peripheral veins is disturbing to many patients. This is the first clinical trial of the efficacy of local anesthesia in comparison to placebo (no pretreatment) in a control group, as a function of the size of the cannula.

Methods: A randomized, controlled trial of fully factorial design was performed to study pain during venipuncture after local anesthesia either with intradermally injected lidocaine or with a vapocoolant spray, in comparison to placebo. A standardized protocol was used for structured communication with the patient to provide the greatest feasible degree of patient blinding (trial registration number DRKS00010155). The primary endpoints were the subjective discomfort of the patient during preparation and puncture of a vein of the dorsum of the hand, assessed on a numerical rating scale (NRS) from 0 (no discomfort) to 10 (unbearable discomfort), and the rate of unsuccessful puncture.

Results: The intention-to-treat analysis of all 450 patients revealed that the reported degree of pain during venipuncture depended to a large extent on the caliber of the chosen venous cannula. For a 17-gauge (17G) cannula, both the vapocoolant spray (NRS $=2.6 \pm 1.3$ ) and lidocaine (NRS $=3.5 \pm 2.2$ ) lessened the discomfort due to venipuncture compared to control treatment $(5.0 \pm 1.5)$. The effect of vapocoolant spray compared to the control was both clinically relevant and statistically significant $(p<0.0001)$. When a smaller $20 \mathrm{G}$ cannula was used, however, vapocoolant spray improved discomfort by only 0.8 NRS points, which, though still statistically significant $(p=0.0056)$, was no longer clinically relevant. The rate of unsuccessful puncture was higher after lidocaine pretreatment $(12.7 \%)$ than after either vapocoolant spray $(4.7 \% ; p=0.0066)$ or no pretreatment $(4.0 \% ; p=0.0014)$.

Conclusion: Local anesthesia can be recommended before venipuncture only if a large cannula is used (e.g., $\geq 17 \mathrm{G}$ ). Vapocoolant spray may be at least as useful as lidocaine injection; it prevents pain to a similar extent and is associated with a lower rate of unsuccessful puncture.

\section{$\checkmark$ Cite this as:}

Rüsch D, Koch T, Spies M, Eberhart LHJ: Pain during venous cannulation-a randomized controlled study of the efficacy of local anesthetics. Dtsch

Arz-tebl Int 2017; 114: 605-11. DOI: 10.3238/arztebl.2017.0605
Clinic of Anesthesiology and Intensive Care Medicine, Philipps-University Marburg: PD Dr. med. Rüsch, Koch, M. Sc., Markus Spies, Prof. Dr. med. Eberhart

UKGM Giessen and Marburg: Markus Spies nserting a cannula into a vein is a routine procedure, which in some patients may none the less cause discomfort $(1,2)$. Surveys, editorials, and systematic reviews on this topic imply that local anesthesia is applied to the venipuncture sites on a regular basis in children, but this is handled rather inconsistently in adults (3-7). In a survey among anesthetists in the UK, the doctors reported that they administered a local anesthetic for venipuncture in cases where the cannula size exceeded 18 G. However, fewer than half of surgeons or specialists in internal medicine followed this practice (6). According to a survey of 71 hospital doctors, $35 \%$ occasionally administered a local anesthetic - mostly lidocaine - before venous cannulation (7). Doctors' reasons for not doing so included the amount of time it takes (45\%), a lacking need/indication $(35 \%)$, and the worry that venous cannulation might be more difficult as a result of this practice $(21 \%)$.

Application of a spray-on cooling agent produces temporary anesthesia at the puncture site. Mixtures of short-chain alkanes are the main substances used in this setting; in the past, various chlorofluorocarbons were also used. Using such vapocoolant sprays before venipuncture has shown inconsistent results in different studies (1, 8-13); methodological reasons do not explain this lack of homogeneity (4).

By contrast, numerous studies have consistently found that intradermal or subcutaneous application of a local anesthetic - for example, lidocaine solution $1-2 \%$-reduces puncture-related pain $(1,8-10,14$, $15)$. A recent network meta-analysis directly or indirectly compared lidocaine application with 16 further analgetic interventions before peripheral venipuncture and found that $2 \%$ lidocaine was most effective (5). However, this procedure is time-consuming (7); it can result in a higher rate of puncture failures $(15,16)$, and of itself causes pain $(8-10,16)$.

It is especially for this reason that the present comparison of different methods aiming to reduce discomfort during venous cannulation does not look at the insertion of the venous cannula in isolation but considers the entire procedure from the moment of applying the tourniquet to the first cannulation attempt. As previous studies rarely considered the importance of the cannula size, we will compare two different cannula sizes in order to determine the optimal pre-treatment of the puncture site. We combined this research question with the rate of failed first attempts at venipuncture. 


\section{Methods}

The study was conducted in 450 inpatients of legal age at Marburg University Medical Center as a monocenter, prospective blinded intervention study with a passive control group (study registration number: DRKS00010155). After arriving in the operating room area, patients having elective surgery were allocated according to instructions from a sealed, nontransparent envelope (randomization on the basis of random numbers from www.random.org) to one of 6 treatment groups consisting of 75 patients each. These comprised all possible combinations (full factorial design) of the two cannula sizes under study (17 Gauge[G] versus $20 \mathrm{G}$ ) and the three options for local pre-treatment of the venipuncture site (intracutaneous injection using $0.1-0.3 \mathrm{~mL}$ lidocaine solution $2 \%$; vapocoolant spray applied for about 2 seconds from a distance of about $5 \mathrm{~cm}$ until the environmental moisture starts forming ice crystals; alcohol-based disinfectant as control). The venipuncture site was always on the dorsum of the hand. During the procedure, doctor and patient communicated in a structured and standardized way, with the patient blinded as well as possible. A third person supervised the blinding (details on the exact proceedings are in the eMethods).

Following the first cannulation attempt, and independently of whether this was successful or unsuccessful, patients rated their level of discomfort caused by the entire venipuncture process (from the moment of applying the tourniquet) on an 11-point numerical rating scale (NRS: $0-10,0=$ no discomfort, $10=$ intolerable discomfort). Taken together with the number of failed attempts at venipuncture, this patient assessment was the primary outcome measure of the study. The NRS discomfort was analyzed initially by using multifactorial analysis of variance (ANOVA) as a global test. If this showed significant between-group differences at the 5\% level, the Tukey-Kramer test was used to assess the effect of the cannula size ( $17 \mathrm{G}$ versus $20 \mathrm{G}$ ) and the local preparation for venipuncture (control versus lidocaine versus vapocoolant spray). The estimated case number was based on the endpoint "failed venipuncture attempt," because - owing to the dichotomous characteristic value-this placed the highest demand in terms of case numbers required. On the basis of older publications $(1,8,10)$, we estimated by using weighted mean values the increase in the rate of failed venipuncture attempts, especially after lidocaine injection, to be 16 percentage points; this estimate was confirmed by a more recent study (16). A two-tailed chi-square test can confirm such a difference (effect size $\omega=0.30$ ) in the setting of a $5 \%$ alpha error and statistical power of $90 \%$ and 141 patients per group, while assuming that the cannula size is an independent factor. Because of expected study dropouts, the group size was increased to 150 patients per intervention. This case number is large enough to confirm differences between groups in the subjective discomfort assessed by the patients (ranked scaled NRS), which is considered as significant upwards at a value of roughly 1 point on the NRS (17-19).

Secondary endpoints of the study were the time required for the venipuncture; this included the respective specific preparation measures taken for administering local anesthesia to the venipuncture site. Furthermore, postoperative skin reactions were documented and described. A total of 5 anesthetists undertook patient recruitment over a time period of 3-6 months respectively. Recruitment started in May 2005 and took 42 months. A second, subsequent study, which investigated pain reduction in cannulation of the radial artery, used similar methods (20).

\section{Results}

Figure 1 shows the distribution of the 450 patients and their allocation to the 6 groups of 75 patients each, as well as the number of and reasons for the 8 cases of non-adherence to the study protocol (flow chart). Because of this small number the results of the perprotocol analysis $(n=442)$ hardly differed from the intention-to-treat analysis $(\mathrm{n}=450)$. Therefore, only the data of the latter is shown. Patients' biometric data and the distribution of the 5 participating doctors were comparable in all groups (eTables 1 and 2).

When patients subjectively rated the discomfort caused by the entire venipuncture procedure, we found that the gauge of the venous cannula used significantly affected patients' ratings, as did the kind of pretreatment of the venipuncture site ( $\mathrm{P}<0.0001$ for both). Figure 2 shows the data as percentiles. The Table shows arithmetic means of all endpoints and frequency rates of failed venipuncture attempts.

\section{Effect of cannula size}

Venipuncture using a $17 \mathrm{G}$ venous cannula was rated across all intervention groups as significantly more uncomfortable than when a small $20 \mathrm{G}$ cannula was used (NRS-17G: $3.8 \pm 2.1$; NRS-20G: $2.7 \pm 1.5 ; \mathrm{P}<0.0001$ ). Differences were greater-2 points - in the control group than in the two active intervention groups (lidocaine: 0.6 ; vapocoolant spray: 0.5 ). The difference between groups in the last two comparisons still reach statistical significance but are no longer clinically relevant.

\section{Effects of local pre-treatment}

When looking at means across all cannula sizes, controls without pre-treatment gave the entire procedure of venipuncture a mean NRS rating of $4.0 \pm 1.7$. A preceding lidocaine injection improved this to $3.2 \pm 2.0$ ( $\mathrm{P}=0.0002$ versus controls). This reduction, however, missed the minimum target of 1 point on the NRS that was defined as clinically relevant. Vapocoolant spray, with a rating of $2.4+/-1.3$ achieved a statistical significant $(\mathrm{P}<0.0001)$ and a clinically relevant improvement when compared to control. Compared with the lidocaine group, the improvement reached statistical significance $(\mathrm{P}=0.0002)$ but was clinically not relevant, with an NRS difference of 0.8 points. 


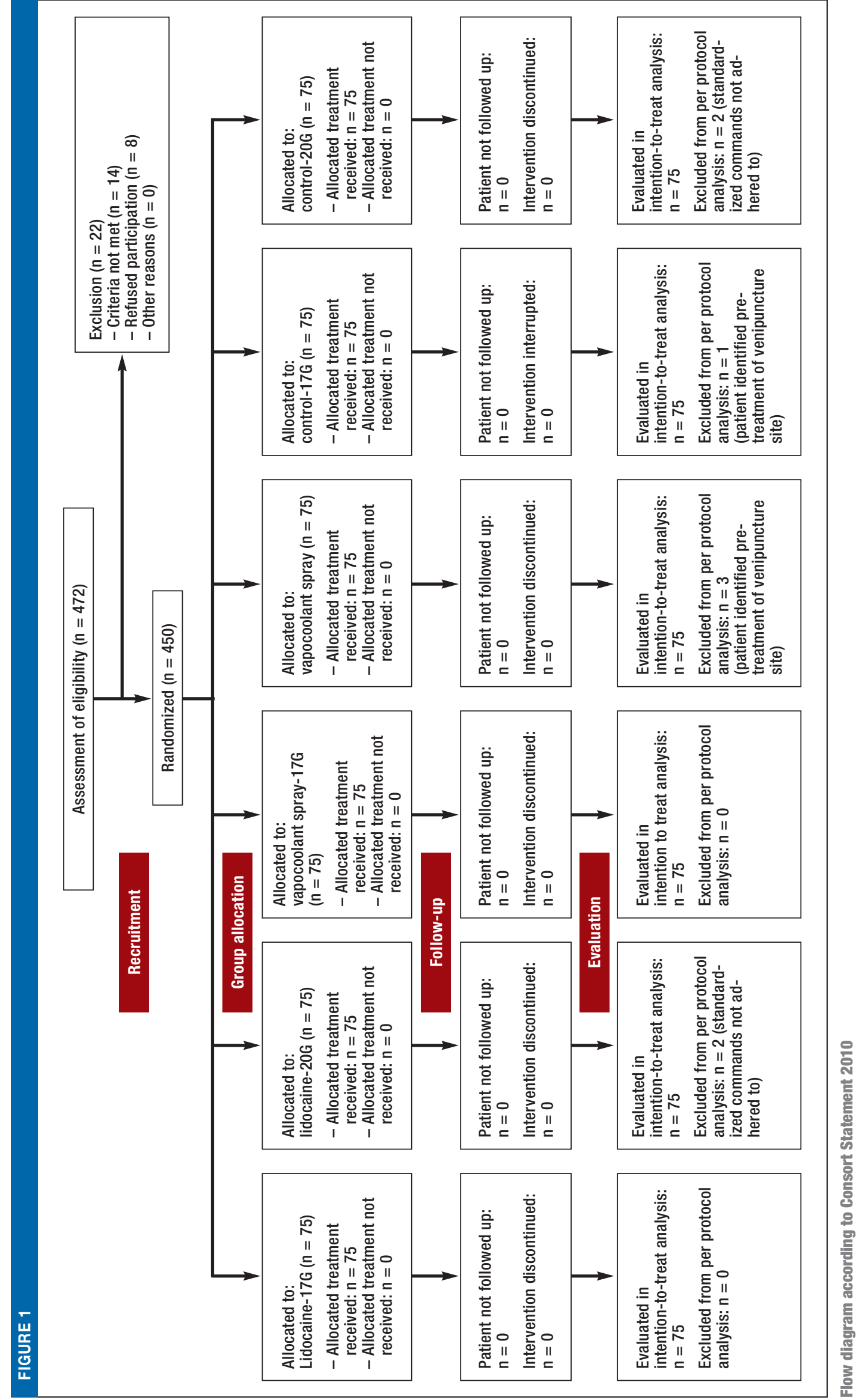




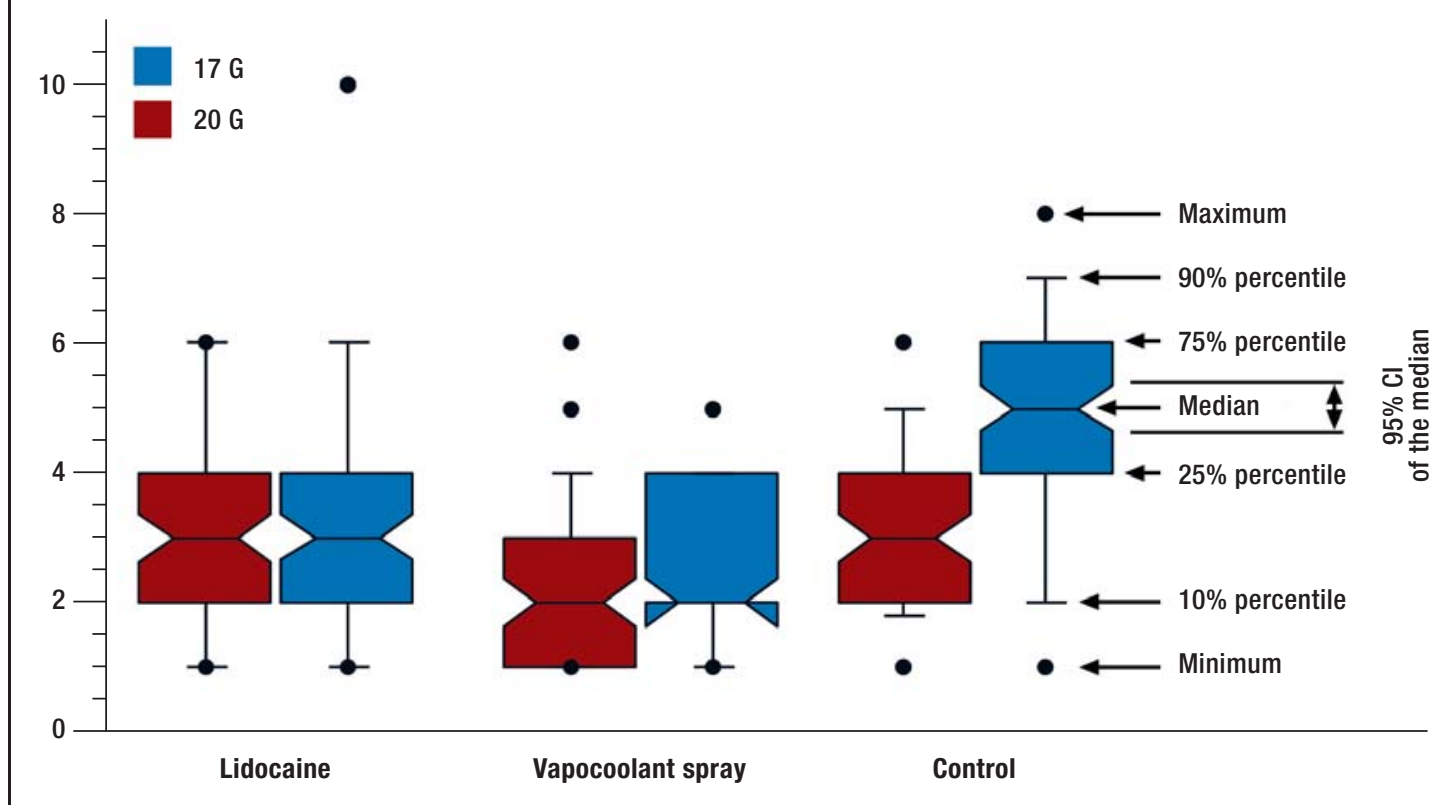

Assessment of subjective discomfort of patients as a result of the venipuncture procedure on an 11-point numerical rating scale from 0 to 10 . $\mathrm{G}$, gauge; $\mathrm{Cl}$, confidence interval

\section{Interactions between pre-treatment and cannula size}

The interaction term of the ANOVA $(\mathrm{P}<0.0001)$ indicated a significant interaction between cannula size and the type of local preparation for venipuncture. Closer analysis and post hoc testing in the subgroups showed that the beneficial effects of both forms of local anesthesia reached statistical significance ( $\mathrm{P}$ values between 0.0001 and 0.0047 ) as well as clinical relevance only when the larger $17 \mathrm{G}$ venous cannulas were used. No significant effects were observed for intradermal or subcutaneous lidocaine application before venipuncture using $20 \mathrm{G}$ venous cannulas. Improvements in patients' subjective ratings of their discomfort after preceding application of vapocoolant spray reached statistical significance $(\mathrm{P}=0.0056)$, but absolute improvement of 0.8 NRS points was too slight to reach genuine clinical relevance.

\section{Failed venipuncture attempts}

Over the course of the study, 32 failed venipuncture attempts occurred (7.1\%). More than half of these $(n=19)$ occurred in the lidocaine group (Table). This accumulation reached statistical significance compared with patients receiving vapocoolant spray $(\mathrm{P}=0.0066)$ or those who did not receive any pre-treatment $(\mathrm{P}=0.0014)$

\section{Process times}

Without any additional preparation of the venipuncture site, the procedure took 50 ( $95 \%$ confidence interval $[48 ; 52])$ seconds, as measured from applying the tourniquet to completion of the venipuncture. Administer- ing vapocoolant spray does not prolong the procedure notably (51 [49; 52] seconds; $\mathrm{P}=0.82)$. In the lidocaine group, however, cannulation times increased on average by approximately half a minute to 75 seconds ([73; 78]; $\mathrm{P}<0.0001$ ) (Table).

\section{Adverse effects}

Except for small hematomata, caused by failed venipuncture attempts or when removing the venous cannula, mild erythema at the venipuncture site was observed in 32 patients in the cooling anesthesia group. In most cases $(n=27)$ this had disappeared by the following day. Only 5 patients experienced skin changes for up to five days. Particular treatment for the observed skin changes was not required.

\section{Discussion}

This clear result regarding the relevance of the size of the venous cannula used enables us to confirm a suspicion that has often been voiced but never consistently shown in studies up to the present. Local anesthetic pre-treatment of the venipuncture site on the dorsum of the hand is indicated when using venous cannulas from a size of $18 \mathrm{G}(6)$. Without such preparation, half of our patients rated the subjective stress/discomfort caused by a $17 \mathrm{G}$ venous cannula to be 5 NRS points or more, $10 \%$ even rated it at 7 points or more. Langham et al. showed even higher scores: 5.7 NRS points for $18 \mathrm{G}$ venous cannulas placed on the dorsum of the hand (21). Further studies observed lower scores (NRS 2.8-4.5), but the puncture site was not defined $(1,11,22)$ or the results were not differentiated by cannula size $(9,22)$. 
Results of primary endpoints from the intention-to-treat analysis ${ }^{* 1}$ and secondary endpoint ${ }^{* 2}$

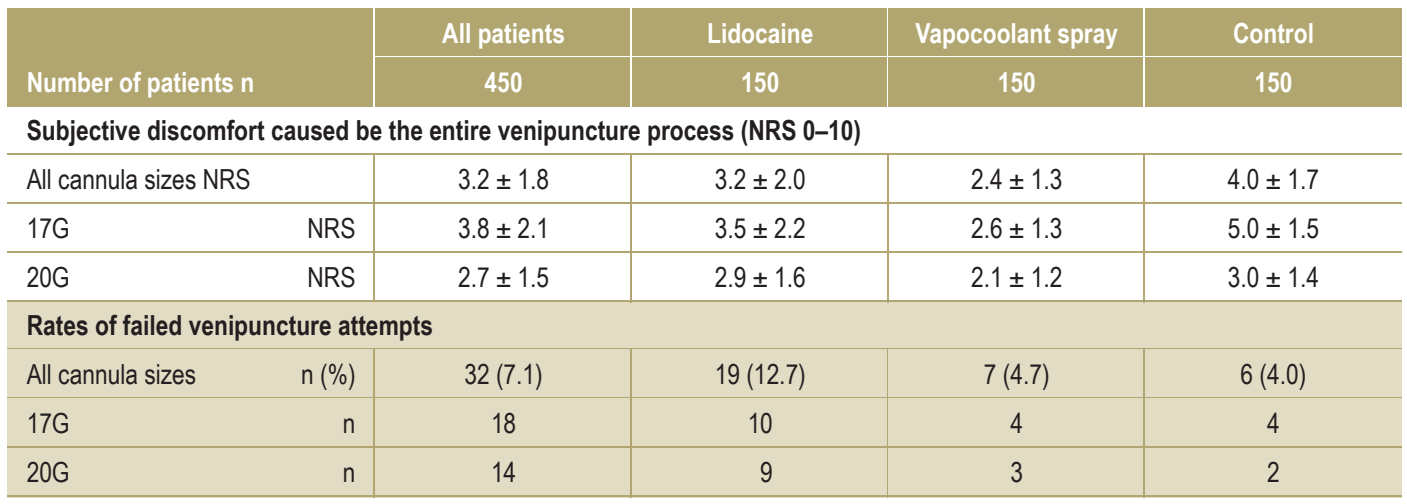

Time expenditure (preparation to completion of venipuncture) in seconds

\begin{tabular}{ll|l|l|l|l}
\hline All cannula sizes & S & $59 \pm 18$ & $77 \pm 15$ & $51 \pm 11$ & $50 \pm 13$ \\
\hline $17 G$ & S & $58 \pm 16$ & $74 \pm 12$ & $50 \pm 11$ & $52 \pm 13$ \\
\hline $20 \mathrm{G}$ & S & $59 \pm 20$ & $79 \pm 16$ & $52 \pm 10$ & $48 \pm 13$
\end{tabular}

\footnotetext{
${ }^{* 1}$ Subjective discomfort caused by the venipuncture procedure and rates of failed venipuncture attempts

${ }^{2}$ Time expenditure for preparation and venipuncture

G, gauge; s, second; NRS, numerical rating scale from 0 (no discomfort) to 10 (intolerable discomfort) as a result of the entire venipuncture process, including preparations; reported as means \pm standard deviations as well as frequencies (percentages)
}

In view of such high levels of pain and impairment/discomfort, patients' scores can be improved by using intradermal lidocaine injection or vapocoolant spray to a statistically significant and clinically relevant degree. Furthermore, it is obvious that patients ${ }^{6}$ stress - and therefore the probable benefit of this intervention-will increase if cannula sizes larger than $17 \mathrm{G}$ are used.

The situation for smaller cannulas (20G and smaller) is very different: The statistically significant effect of cryoanesthesia seems clinically not relevant, as it is 0.75 points on the NRS and thus just misses the measuring method's ability to discriminate $(17,18)$. This result is consistent with the results of a meta-analysis of the benefits of cryoanesthesia before venipuncture (3). Across all studies, venipuncture using smaller cannula sizes $(22 \mathrm{G}$ and smaller) (10/100 points) reduced pain in children, but did not reach statistical significance. With the exception of one study (9), exclusively $20 \mathrm{G}$ venous cannulas were used in adults as well. Compared with one venipuncture without pre-treatment, the meta-analysis found a pooled reduction in pain at the 10/100 level; because of a larger total number of cases than in the preceding comparison, this reached statistical significance (3). Compared with genuine placebo treatment, the pain reduction of $12 / 100$ points did not reach significance any longer. A limitation of this meta-analysis is the pooling of studies in which different sizes of venous cannulae were used for the purpose of venipuncture in different areas of the body. Furthermore, the range of different vapocoolant sprays used was wide. On the face of it it may seem irrelevant which substances were used to produce the evaporation chill. However, it is crucial to hit the desired venipuncture site precisely by using a brief puff of the spray. Different preparations differ widely in this respect, as they were mostly conceived for use on larger areas of the body, for example, for joints.

The question of which method yields better results before inserting a $17 \mathrm{G}$ (or larger) venous cannula cannot be decided on the basis of patients' ratings alone. In the direct comparison, vapocoolant is superior to lidocaine injection $(\mathrm{P}=0.0047)$, but the difference of 0.9 NRS points fails ever so slightly the criterion for clinical relevance. The results are therefore comparable with those from a follow-up study of the cannulation of the radial artery (20). After intradermal or subcutaneous injection of lidocaine, the rate of failed venipuncture attempts increased significantly and more than doubled - probably because of the poorer differential of the vein's contour. Overall, the outcome is in favor of cryoanesthesia, especially as it is easy to conduct and no long-term adverse effects have been confirmed if it is applied correctly.

\section{Limitations of the study}

An additional aspect that causes lidocaine injection to appear in a less favorable light is the methodological handling of a failed venipuncture. The affected patients gave their scores before the required follow-up puncture, which might have had a negative influence on the overall assessment.

Furthermore, the lack of genuine blinding of patients is a central problem of this study, which is also the case for all other, comparable studies. In most studies, patients were asked to close their eyes during the venipuncture process, which entails the risk of de-blinding in the truest 
sense of the word. In our study, we tried to minimize this risk by using a neutral observer. This observer ensured during the entire procedure that the patients were neither able to see the preparations nor the procedure itself, and that standardized communication was adhered to. This helped identify a protocol violation in 8 patients (Figure 1). Adding a third group (vapocoolant spray) increased the challenge of concealing the type of intervention from patients. We used strictly standardized communication with patients to inform them about the application of vapocoolant spray or the application of alcohol-based disinfectant spray without mentioning which of the two was actually applied. The lidocaine injection and the actual venipuncture were also announced using the exact identical wording, again without unveiling which of the two was actually applied. However, patients in whom vapocoolant spray had been used in the past would have been able to distinguish the less intensive sensation of cold owing to the evaporation of alcohol from the strong cooling sensation of the alkane spray. Ultimately, as in comparable studies, it remains unclear whether the efforts involved in blinding patients to their treatment were actually successful.

In principle, patients who think they are in an intervention group can be expected to be more prone to making a better judgement of the subjective discomfort caused by the venipuncture. This should be borne in mind when interpreting the data.

The size selection of the venous cannulas used is based on the fact that in our hospital, $18 \mathrm{G}$ cannulas are used most often, according to data from the local resource/materials management. The selection of a larger or smaller caliber was intended to improve the results' ability to discriminate. $17 \mathrm{G}$ and $20 \mathrm{G}$ are therefore representative for "large" and "small" cannula diameters.

\section{Conclusions}

The present results underline the indication for local anesthetic pre-treatment if a venous cannula of $17 \mathrm{G}$ or larger is inserted on the dorsum of the hand. Cryoanesthesia may offer advantages in this setting, compared with the thus far more common lidocaine infiltration, in terms of condition of the puncture site, effectiveness, and simplified processes. In smaller venous cannulas (20G and smaller), positive effects are statistically significant. However, these are small and clinically not relevant.

\section{Conflict of interest statement}

The authors declare that no conflict of interest exists.

Manuscript received on 13 0ctober 2016, revised version accepted on 12 June 2017

Translated from the original German by Birte Twisselmann, PhD.

\section{REFERENCES}

1. Biro P, Meier T, Cummins, AS: Comparison of topical anaesthesia methods for venous cannulation in adults. Eur J Pain 1997: 1: 37-42.
KEY MESSAGES

- When larger cannulas are used-for example 17G_-both intradermal injection of lidocaine and vapocoolant spray prior to cannulation reduce venipuntureassociated adverse effects to a clinically and statistically significant extent.

- Where smaller-gauge cannulas (such as $20 \mathrm{G}$ ) are used, patients' subjective discomfort is comparatively low and the pre-treatment interventions did not yield any clinically relevant improvements.

- While vapocoolants increase the rate of failed venipuncture attempts and the time of the process only slightly, intradermal injection of lidocaine results in significantly higher rates of failed venipuncture attempts and significantly longer process times compared with placebo.

- Because of the benefits mentioned above and lower use of resources in peripheral venipuncture, with comparable effectiveness, cryoanesthesia can be considered as an at least equal alterative to intradermal lidocaine application.

2. Speirs AF, Taylor KH, Joanes DN, Girdler NM: A randomised, doubleblind, placebo-controlled, comparative study of topical skin analgesics and the anxiety and discomfort associated with venous cannulation. Br Dent J 2001; 190: 444-9.

3. Hogan ME, Smart S, Shah V, Taddio A: A systematic review of vapocoolants for reducing pain from venipuncture and venous cannulation in children and adults. J Emerg Med 2014; 47: 736-49.

4. Moore AS, Straube S, McQuay H: Minimising pain during intravenous cannulation. Br Med J 2009; 338: a2993.

5. Bond M, Crathorne L, Peters J, et al.: First do not harm: pain relief for the peripheral venous cannulation of adults, a systematic review and network meta-analysis. BMC Anesthesiology 2016; 16: 81.

6. Sado DM, Deakin CD: Local anaesthesia for venous cannulation and arterial blood gas sampling: are doctors using it? J R Soc Med 2005; 98: 158-60.

7. Norris WD: The use of local anaesthetics in peripheral venous cannulation: current practise of junior doctors. J R Nav Med Serv 2002; 88: 62-4.

8. Armstrong P, Young C, McKeown D: Ethyl chloride and venepuncture pain: a comparison with intradermal lidocaine. Can J Anaesth 1990; 37: 656-8.

9. Robinson PA, Carr S, Pearson S, Frampton C: Lignocaine is a better analgesic than either ethyl chloride or nitrous oxide for peripheral intravenous cannulation. Emerg Med Australas 2007; 19: 427-32.

10. Selby IR, Bowles BJ: Analgesia for venous cannulation: a comparison of EMLA (5 minutes application), lignocaine, ethyl chloride, and nothing. J R Soc Med 1995; 88: 264-7.

11. Hartstein BH, Barry JD: Mitigation of pain during intravenous catheter placement using a topical skin coolant in the emergency department. Emerg Med J 2008; 25: 257-61.

12. Costello M, Ramundo M, Christopher NC, Powell KR: Ethyl vinyl chloride vapocoolant spray fails to decrease pain associated with intravenous cannulation in children. Clin Pediatr 2006; 45: 628-32.

13. Farion KJ, Splinter KL, Newhook K, Gaboury I, Splinter WM: The effect of vapocoolant spray on pain due to intravenous cannulation in children: a randomized controlled trial. CMAJ 2008; 179: 31-6. 
14. Harris T, Cameron PA, Ugoni A: The use of pre-cannulation local anaesthetic and factors affecting pain perception in the emergency department setting. Emerg Med J 2001; 18: 175-7.

15. Hendry F, Checketts MR, McLeod GA: Effect of intradermal anaesthesia on success rate and pain of intravenous cannulation: a randomized non-blind crossover study. Scott Med J 2011; 56: 210-3.

16. Page DE, Taylor DM: Vapocoolant spray vs subcutaneous lidocaine injection for reducing the pain of intravenous cannulation: a randomized, controlled, clinical trial. Br J Anaesth 2010; 105: 519-25.

17. Todd KH, Funk KG, Funk JP, Bonacci R: Clinical significance of reported changes in pain severity. Ann Emerg Med 1996; 27: 485-9

18. Kelly AM: Does the clinically significant difference in visual analog scale pain scores vary with gender, age, or cause of pain? Acad Emerg Med 1998; 5: 1086-90.

19. Gallagher EJ, Liebmann M, Bijur PE: Prospective validation of clinically important chances in pain severity measured on a visual analog scale. Ann Emerg Med 2001; 38: 633-8.

20. Rüsch D, Koch T, Seel F, Eberhart L: Vapocoolant spray versus lidocaine infiltration for radial artery cannulation: a prospective, ran- domized, controlled clinical trial. J Cardiothorac Vasc Anesth 2017; 31: 77-83.

21. Langham BT, Harrison DA: Local anaesthetic: does it really reduce the pain of insertion of all sizes of venous cannula? Anaesthesia 1992; 47: 890-1.

22. Hijazi R, Taylor D, Richardson J: Effect of topical alkane vapocoolant spray on pain with intravenous cannulation in patients in emergency departments: randomised double blind placebo controlled trial. BMJ 2009; 338: b215.

\section{Corresponding author}

Prof. Dr. med. Leopold Eberhart

Klinik für Anästhesie und Intensivtherapie

Baldingerstr., 35033 Marburg, Germany

Supplementary material

For eReferences please refer to: www.aerzteblatt-international.de/ref3717

eMethods, eTables:

www.aerzteblatt-international.de/17m0605

\section{CLINICAL SNAPSHOT}

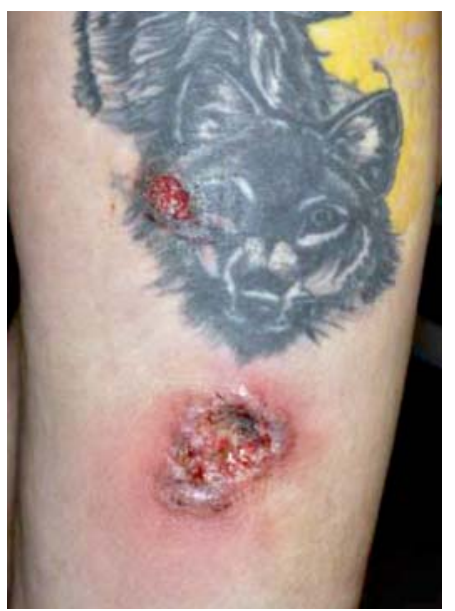

\section{Pyoderma Gangrenosum after a Biopsy}

A 33-year-old woman chronically suffering from ulcerative colitis complained of very painful skin changes on her thigh and was referred with a putative diagnosis of cowpox. Inspection revealed two wounds, each $1 \mathrm{~cm}$ in diameter, surrounded by pus and erythema. Serological tests and bacteriological and virological culture swabs were obtained, and a tissue biopsy was performed. One week later, despite local antiseptic treatment, the lesions had become larger and extremely painful. The serological tests and bacterial and viral cultures yielded no clear diagnosis. Histopathological examination revealed inflammation with many neutrophils.

The diagnosis of pyoderma gangrenosum was made in consideration of all the findings and after the exclusion of relevant differential diagnoses. Systemic glucocorticoid treatment was initiated, and the patient's pain improved promptly and markedly; the lesions healed completely, with scarring, in two months' time. In this case, the biopsy led to a rapid increase in the size of the lesions-an illustration of the so-called pathergy phenomenon.

Prof. Dr. med. Joachim Dissemond, PD Dr. med. Andreas Körber

Klinik und Poliklinik für Dermatologie, Venerologie und Allergologie, Universitätsklinikum Essen

joachim.dissemond@uk-essen.de

\section{Conflict of interest statement}

The authors state that they have no conflict of interest.

\section{Cite this as:}

Dissemond J, Körber A: Pyoderma gangrenosum after a biopsy. Dtsch Arztebl Int 2017; 114: 611.

DOI: 10.3238/arztebl.2017.0611

Translated from the original German by Ethan Taub, M.D. 


\section{Supplementary material to:}

\section{Pain During Venous Cannulation}

A Randomized Controlled Study of the Efficacy of Local Anesthetics

by Dirk Rüsch, Tilo Koch, Markus Spies, and Leopold HJ. Eberhart

Dtsch Arztebl Int 2017; 114: 605-11. D0I: 10.3238/arztebl.2017.0605

\section{eREFERENCES}

e1. Böhmer AB, Wappler F, Zwissler B: Perioperative risk assessment-from routine tests to individualized investigation. Dtsch Arztebl Int 2014; 111: 437-46.

e2. Schlitt A, Jámbor C, Spannagl M, Gogarten W, Schilling T, Zwissler $\mathrm{B}$ : The perioperative management of treatment with anticoagulants and platelt aggregation inhibitors. Dtsch Arztebl Int 2013; 110: 525-32.

e3. Torossian A, Bräuer A, Höcker J, Bein B, Wulf H, Horn EP: Preventing inadverent perioperative hypothermia. Dtsch Arztebl Int 2015: 112: 166-72.

e4. Evans JG, Taylor DM, Hurren F, Ward P, Yeoh M, Howden BP: Effects of vapocoolant spray on skin sterility prior to intravenous cannulation. J Hosp Infect 2015; 90: 333-7. 


\section{How the study was conducted}

The present study received ethics approval from the ethics committee at the Faculty of Medicine, Philipps-University Marburg (Ref 103/02). At the end of the recruitment period it was entered in the German Clinical Trials Register (Deutsches Register für klinische Studien, www.drks.de; registration number: DRKS00010155). The study was funded by the Department of Anesthesia and Intensive Care Therapy, Philipps-University Marburg.

Patients who met all inclusion criteria and to whom none of the exclusion criteria applied received written and oral information and were then invited on the day before their operation to give their written consent to the planned study. The following inclusion criteria were defined:

- Elective surgical procedure

- Planned venipuncture before administration of anesthesia on the dorsum of the hand, examination of the vein found it in sufficiently good condition for a $17 \mathrm{G}$ venous cannula to be inserted

- No language barrier - able to communicate in German

- Patient's cognitive performance is unimpaired

Exclusion criteria:

- Raynaud's disease

- Lidocaine intolerance or allergies

- Skin changes, such as hematomata, scars, rashes, or infections on the dorsum of the hand.

Patients were included in the study over five time periods of 3-6 months, starting in May 2005. Recruiting 450 patients took a total of 42 months. Five anesthetists in sequence undertook the venous cannulation.

On the evening before and the morning of their surgery, patients received oral premedication of dipotassium clorazepate $20 \mathrm{mg}$. Patients ' anesthesiologic preparation and continuation of patients' own medication followed current recommendations (e1, e2). After arriving in the anesthesia preparation area, routine monitoring measures were initiated-consisting of electrocardiography, pulse oximetry, and oscillometric blood pressure monitoring. Patients were warmed up in order to prevent intraoperative hypothermia (e3).

The following items were made readily available for each patient:

- One venous cannula each of $17 \mathrm{G}$ (white) and $20 \mathrm{G}$ (pink)

- Alcohol-based disinfectant spray containing ethanol and isopropanol

- Vapocoolant spray (alkane mixture consisting of propane butane, and pentane)

- $0.5 \mathrm{~mL}$ of lidocaine $2 \%$ solution in a $2 \mathrm{~mL}$ syringe with $27 \mathrm{G}$ needle attached

- Alcohol-based disinfectant swabs

- I.V. dressing

According to the scheduled procedure, the side of the venipuncture was decided on and the material was prepared on that side. In order to collect data on the duration of the subsequent intervention, the time was measured after the preparations had been concluded (collating the materials and connecting the patients to the monitors). Patients were asked to turn their head to the side opposite the site of the venipuncture and to focus their gaze there, in order to prevent them from observing the procedure. A third observer monitored the patient's direction of gaze during the subsequent procedure and also the standardized communication of the doctor performing the venipuncture with the patient. After placing the tourniquet, the anesthetist identified a vein on the dorsum of the patient's hand. This site was disinfected by using and alcohol-impregnated swab, so as to ensure the hygienic preparation of the venipuncture site (e4). Only then, immediately before the following measures, did the anesthetist open an envelope (sealed, non-transparent), which included information on the patient's group allocation. Local anesthetic pre-treatment of the venipuncture site (vapocoolant spray, lidocaine injection, no further measure as control group) and the size of the venous cannula were defined in accordance with the factorial study design. Randomization had been done before the actual study, by staff not involved in the venipuncture, who used random numbers (www.random.org).

The subsequent communication with the patient was bound by strict rules. After the randomization envelope had been opened, the following announcement was addressed to the patient: „You will shortly feel cold on the dorsum of your hand." Patients in the lidocaine group and the control group then had alcohol-based disinfectant sprayed on to the dorsum of their hand. Patients in the cryoanesthesia group, by contrast, received a puff of the freezing spray. This was administered from a distance of about $5 \mathrm{~cm}$, until the venipuncture site was covered in a white mix of frozen moisture from skin and environmental air. The duration was $1-2$ seconds, with a maximum of 3 seconds.

After the cold stimulant had been applied (alcohol spray or vapocoolant spray), the next standardized communication to the patient was issued: „I am now starting the venipuncture.“ In patients in the cryoanesthesia group and the control group, the venipuncture was started then. Patients in the lidocaine group, however, first received an intradermal injection of $0.1-0.3 \mathrm{~mL}$ of the lidocaine $2 \%$ solution by using the $27 \mathrm{G}$ syringe. Immediately afterwards, venipuncture was undertaken and the venous cannula inserted, but no further announcement was made.

When the first venipuncture had been completed, the time was stopped, independently of whether the procedure had been successful or not. Before an i.v. dressing was applied - that is, while remaining ignorant about the success of the procedure-patients were invited to rate their subjective discomfort throughout the entire procedure. During the initial consultation, this approach had been discussed with the patient, and the use of the 11-point numerical rating scale (NRS) had been practiced. This was intended to ensure that all problems noted during the preparations-for example, tightness of the tourniquet, impact of the cold and burning pain, but also pain at the venipuncture site-were included in the overall assessment.

The invitation to participants to rate their experience using the NRS was communicated by using a standardized phrase: „Please rate how uncomfortable the venipuncture procedure was for you just now. Pick a number from 0 - no discomfort - up to 10 for intolerable discomfort." Only after the rating had been performed, the i.v. dressing was applied or another cannulation attempt was initiated where required. 
All patients were followed up with regard to local adverse effects of the venipuncture or the preparations undertaken to this end until they were discharged from hospital.

\section{Study objective and clinical endpoints}

The study aimed to investigate the effects of two methods of local anesthetic pre-treatment of the venipuncture site before inserting venous cannulas of different sizes. On the one hand, the focus was on evaluating the entire proceedings of the venipuncture (including prepping the patient) and, on the other hand, on the result of the venipuncture. The two aspects cannot be separated as a venipuncture - even if not experienced as stressful by a patient - is always accompanied by additional discomfort if it has to be repeated because it failed the first time. Therefore, both aspects (subjective discomfort of the patient and the rate of failed venipuncture attempts) were equal primary endpoints of the study. The patients scored their experience in an 11-point NRS from $0-10$; rates of failed venipuncture attempts were collected by means of documenting of the venipuncture result. Process times (duration from applying the tourniquet to the completion of the first venipuncture attempt) as well as possible local adverse effects of the local/cryoanesthesia were secondary endpoints.

\section{Sample size calculation}

Of the two primary endpoints, the rate of failed venipuncture attempts as a dichotomous endpoint necessitates a greater requirement for an adequate number of participants, and the sample size calculation was therefore based on this. Based on older publications $(1,8,10)$ the researchers estimated on the basis of weighted means the increase in the rate of failed venipuncture attempts, especially after lidocaine injection, at 16 percentage points, which was confirmed by a subsequent study (16). A two-tailed chi-square test can be used to confirm such a difference (effect size $\omega=0.30$ ) for an alpha error of $5 \%$ with a power of $90 \%$ and 141 patients per group, if independence of the cannula size is assumed and therefore the failed venipuncture rates for both cannula sizes are combined. Because of expected dropouts the sample size was increased to 150 patients per intervention. The large number of 450 patients made it possible with regard to the second primary endpoint to detect small scoring differences on the NRS in the range of 1.27 points. This assumes that the standard deviation of the NRS scores is twice as high as the difference between groups. The Tukey-Kramer (all pair) test achieves a power of $90 \%$ in this setting and also allows a Bonferroni correction of the alpha error to $2.5 \%$. Consequently, the entire study was of sufficient power to verify the results of earlier studies with regard to the rate of failed venipuncture attempts and simultaneously show these as a clinically relevant improvement in patients" scores on the NRS. Sample size calculation was performed using PASS 2002

\section{Definition of clinical relevance}

In different studies, NRS differences between 0.9 and 1.3 points were defined as clinically relevant (17-19) on a visual analog scale (VAS) of $10 \mathrm{~cm}$. In the named studies, this is the order of magnitude of patients' ability to discriminate a pain sensation as slightly lower or slightly higher than in a reference stimulus by using this instrument. For the clinical interpretation of the results, the results on the VAS were applied to the NRS used in this study, to simplify the results; a difference of one NRS point was defined as clinically relevant.

\section{Statistical evaluation}

The plan for the statistical evaluation prospectively set out in the study protocol comprised the global analysis of the rate of failed venipuncture attempts with a chi-square test between the 3 therapeutic groups (cryoanesthesia, lidocaine injection, and controls). In case of a significant effect at the 5\% level, Fisher's exact test would then be used as a post hoc testing method to look for the source of the unequal cell frequencies. In the patients' evaluation using the NRS, the initial step was to globally search for differences between the 3 treatment groups and the influence of the cannula size by using a three-factorial analysis of variance (ANOVA). Where the significance level adjusted to $2.5 \%$ had not been reached, the Tukey-Kramer (all pair) test was used for the specific effects of the cannula sizes on the one hand and the pre-treatment of the venipuncture site on the other hand. Secondary endpoints were analyzed descriptively. In order to take into consideration that the NRS scores are merely rank scaled data, Figure 2 shows the box plots of the scores and the Table the corresponding arithmetic means and standard deviations. The software package JMP 9.0.1 (SAS-Institute, Cary, NC, USA 27513) was used for all statistical analyses. 


\section{eTABLE 1}

Demographic and biometric patient data

\begin{tabular}{|l|c|c|c|c|}
\hline \multirow{2}{*}{ Number of patients $n$} & All patients & Lidocaine & Vapocoolant & Control \\
\cline { 2 - 5 } & 450 & 150 & 150 & 150 \\
\hline
\end{tabular}

$\operatorname{Sex}(m: f)$

\begin{tabular}{lr|r|r|r|l}
\hline All cannula sizes & & $194: 256$ & $65: 85$ & $63: 87$ & $66: 84$ \\
\hline $17 \mathrm{G}$ & $\mathrm{n}$ & $99: 126$ & $30: 45$ & $34: 41$ & $35: 40$ \\
\hline $20 \mathrm{G}$ & $\mathrm{n}$ & $95: 130$ & $35: 40$ & $29: 46$ & $31: 44$ \\
\hline Age & & & & \\
\hline All cannula sizes & years & $49.6 \pm 16.8$ & $49.5 \pm 15.9$ & $49.0 \pm 18.0$ & $51.0 \pm 16.3$ \\
\hline $17 \mathrm{G}$ & years & $50.1 \pm 16.4$ & $47.8 \pm 17.6$ & $48.7 \pm 15.4$ & $54.1 \pm 15.5$ \\
\hline $20 \mathrm{G}$ & years & $49.2 \pm 17.3$ & $51.1 \pm 14.0$ & $49.2 \pm 20.5$ & $47.9 \pm 16.7$
\end{tabular}

Data are shown as frequencies or means \pm standard deviations.

\section{eTABLE 2}

Distribution of doctors undertaking venipuncture in the 6 intervention groups

\begin{tabular}{|c|c|c|c|c|c|c|}
\hline Cannula size/intervent & & Doctor 1 & Doctor 2 & Doctor 3 & Doctor 4 & Doctor 5 \\
\hline $20 \mathrm{G} /$ no intervention & $\mathrm{n}(\%)$ & $13(2.9)$ & $15(3.3)$ & $15(3.3)$ & $20(4.4)$ & $12(2.7)$ \\
\hline 20 G/freeze spray & $\mathrm{n}(\%)$ & $13(2.9)$ & $19(4.2)$ & $16(3.6)$ & $22(4.9)$ & $5(1.1)$ \\
\hline 20 G/lidocaine & $\mathrm{n}(\%)$ & $11(2.4)$ & $16(3.6)$ & $17(3.8)$ & $20(4.4)$ & $11(2.4)$ \\
\hline $17 \mathrm{G} /$ no intervention & $\mathrm{n}(\%)$ & $9(2.0)$ & $18(4.0)$ & $20(4.4)$ & $20(4.4)$ & $7(1.6)$ \\
\hline 17 G/vapocoolant spray & & $9(2.0)$ & $18(4.0)$ & $14(3.1)$ & $23(5.1)$ & $11(2.4)$ \\
\hline 17 G/lidocaine & $\mathrm{n}(\%)$ & $13(2.9)$ & $19(4.2)$ & $18(4.0)$ & $19(4.2)$ & $6(1.3)$ \\
\hline
\end{tabular}

Data are shown as absolute and proportional (in parentheses) frequencies. 